\title{
Audit of Patients with Multiple Endocrine Neoplasia Type 1 (MEN1): Screening of Pancreatic Neuroendocrine Tumours (pNETs), Parathyroid Tumours and Pituitary Adenomas
}

MP Kyithar', L Cullen², CS Lee², N Swan², J Geoghegan², S Skehan², R Crowley'1,2, D O’Shea1,2, D O'Toole ${ }^{2}$

St. Vincent's Healthcớre

1Endocrinology Department, St. Vincent's University Hospital, Dublin, Ireland

${ }^{2}$ Neuroendocrinology Multidisciplinary Team, St. Vincent's University Hospital, Dublin, Ireland

\section{INTRODUCTION}

Patients with MEN1 have increased morbidity and mortality compared to those patients with sporadic NETs.

No genotype-phenotype correlation is described and age-related clinical penetrance surpasses $50 \%$ and $90 \%$ by 20 and 40 years, respectively.

The aim of the audit was to compare the screening programme for MEN1 patients with MEN1 clinical guidelines.

\section{METHODS}

Case notes of MEN1 patients attending a tertiary NET-multidisciplinary team (MDT) in Ireland were reviewed.

All patients attending the NET-MDT have gastrointestinal hormone, parathyroid, pituitary profiles, endoscopic and imaging studies according to guidelines.

\section{RESULTS}

\section{Patients with MEN1}

-13 patients (11 kindreds), 100\% Caucasians, mean age 43 years (range 28-67): 69\% male, 31\% female -85\% $(n=11)$ had confirmed MEN1 mutations and two had clinical or familial MEN1

\section{Reasons for Patients referred to NET-MDT}

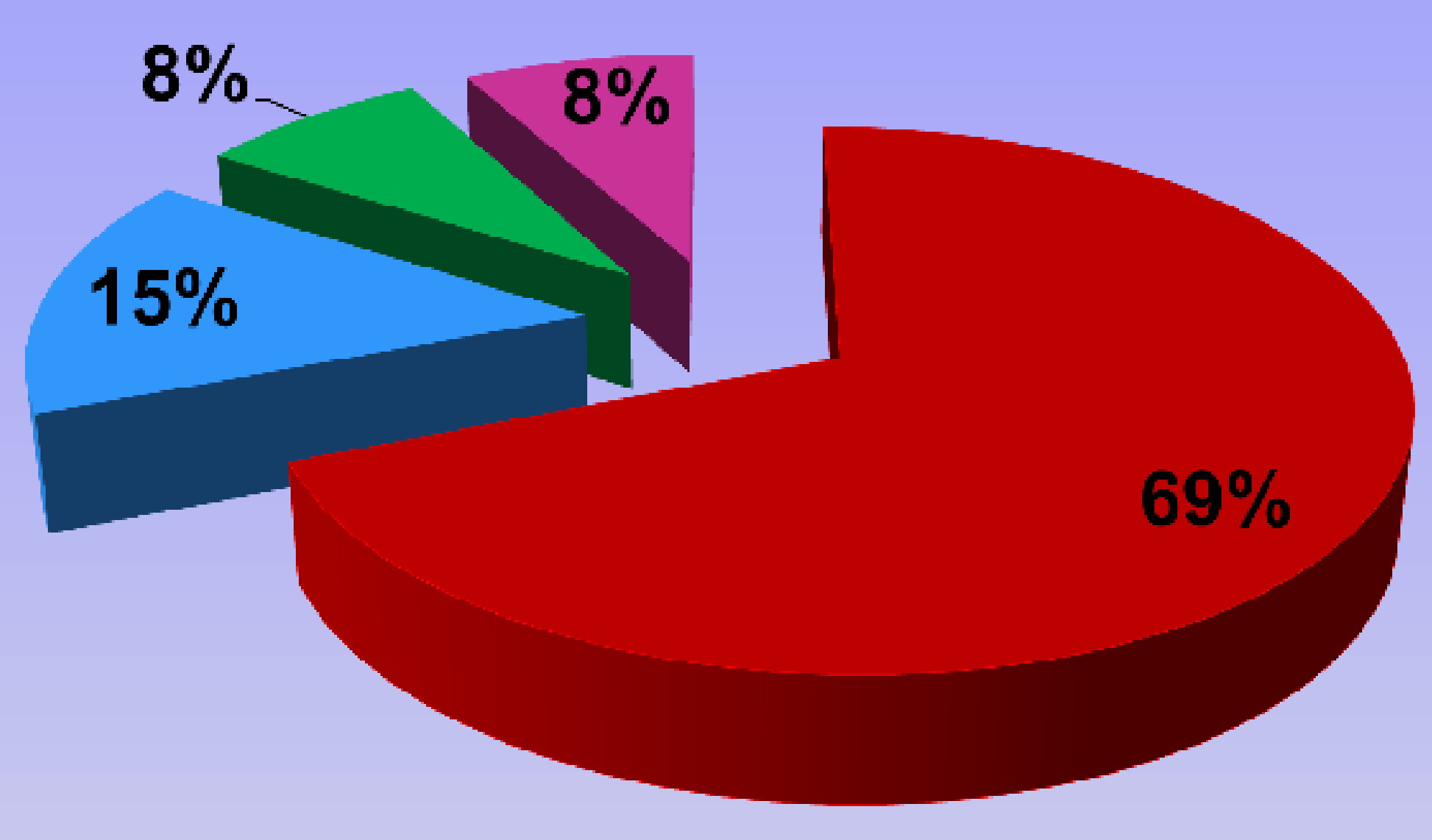

- Screening for known

MEN1 mutations

Family screening

Management of pNETs

- Screening due to PHPT \& acromegaly
Prior to NET-MDT assessment,

- $46 \%$ did not have endoscopic/radiology studies to screen for pNETs

- none of the patients had CT/MRI thorax to screen for thymic/bronchial NETs

Age at screening

- age referred to NET-MDT: $41.5 \pm 12.2$ years.

- age at endoscopic/radiological screening for NETs (prior to/at NET-MDT): $37.1 \pm 14.3$ years

- age at diagnosis of NETs: $34.9 \pm 14.3$ years

On screening of NET-MDT, the following new diagnoses were made:

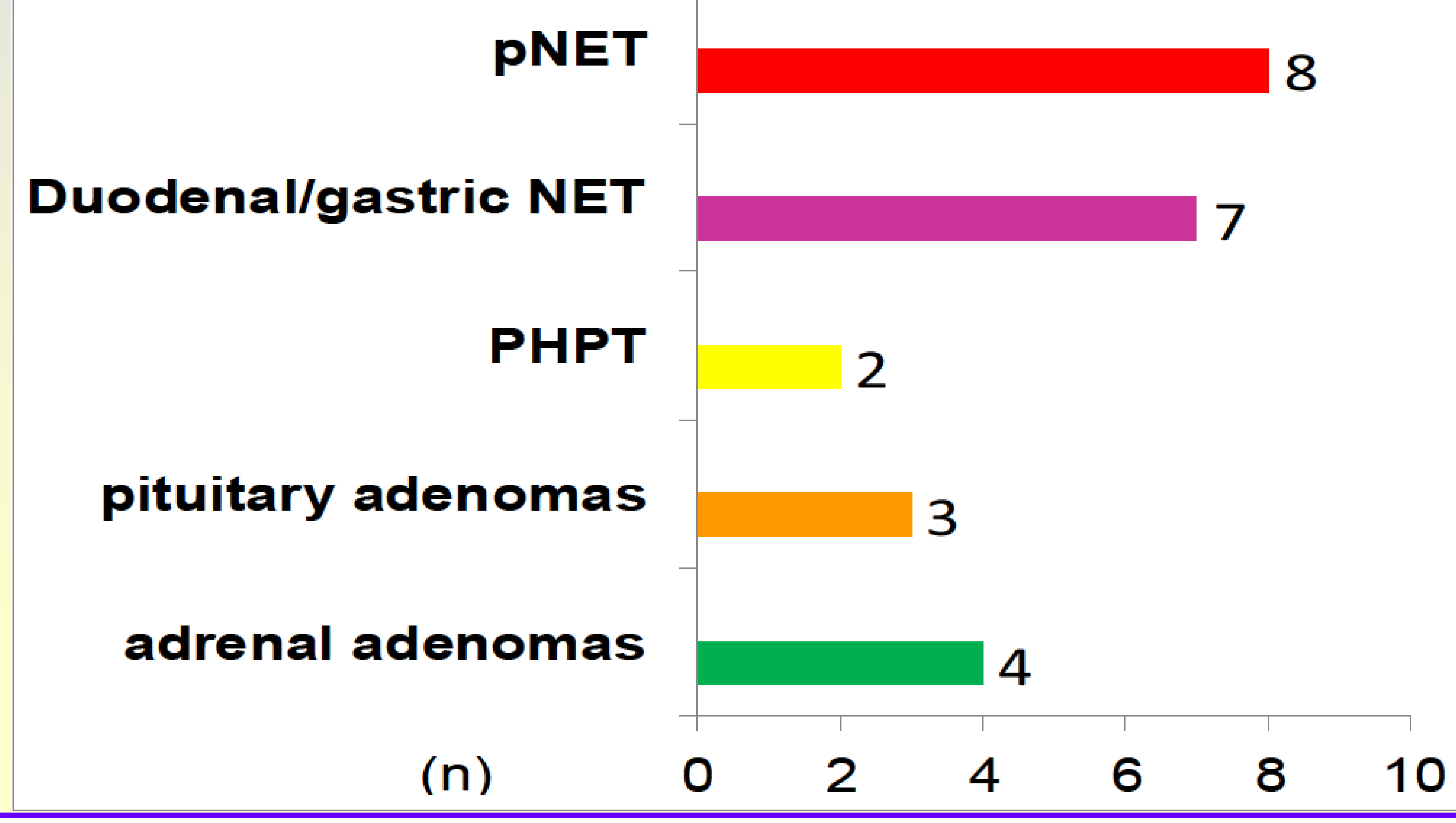

\section{CONCLUSIONS}

References

Endoscopic/radiological screening of NETs occurred at later age than recommended by current guidelines.

Surveillance methods were also largely at variance with guidelines.

Referral to a dedicated MDT has identified a significant number of previously unrecognised neuroendocrine pathologies. 